\title{
Erratum to: Phase Correction Using Programmable Phase Modulator (PPM) in Optical Coherence Tomography
}

\author{
Muhammad Faizan Shirazi, Woonggyu Jung and Jeehyun Kim
}

(C) The Korean Society of Medical \& Biological Engineering and Springer 2014

In the volume 4, issue 1 of the Biomedical Engineering Letters, an error occurred in the research article:

Muhammad Faizan Shirazi, Woonggyu Jung and Jeehyun Kim

Phase Correction Using Programmable Phase Modulator (PPM) in Optical Coherence Tomography

Biomed Eng Lett. (2014) 4:77-85 DOI 10.1007/s13534-0140119-1
- In the page number

Original wording:

Biomed Eng Lett (2014) 4:77-85

This should be replaced by:

Biomed Eng Lett (2014) 4:64-72

Muhammad Faizan Shirazi, Jeehyun Kim $(\bowtie)$

School of Electronics Engineering, Kyungpook National University,

Daegu, Korea

Tel : +82-53-950-7221/ Fax : +82-53-959-7221

E-mail : jeehk@knu.ac.kr

Woonggyu Jung

School of Nano-Bioscience \& Chemical Engineering, Ulsan National Institute of Science and Technology, Ulsan, Korea 